Abstract P202 Table 1 Multidomain assessment for children before and after 6-month mepolizumab trial and 4-month omalizumab trial

\begin{tabular}{|c|c|c|c|c|}
\hline Domain assessed & & Mepolizumab & Omalizumab & $\begin{array}{l}\text { Comparison between } \\
\text { biologics: } \\
\text { P-value }\end{array}$ \\
\hline \multirow[t]{4}{*}{ Monthly exacerbation rate } & Baseline & $\begin{array}{l}0.333(0.167- \\
0.792) n=12\end{array}$ & $0.75(0.5-1) n=31$ & 0.218 \\
\hline & End of biologics trial & $0(0-0.333) n=12$ & $0.25(0-0.5) n=31$ & \\
\hline & $\begin{array}{l}\text { Paired comparison of baseline and end } \\
\text { of trial P-value }\end{array}$ & 0.234 & $0.0001 * * *$ & \\
\hline & Median (IQR) change/person & $-0.167(-0.583-0)$ & $-0.5(-0.5-0)$ & 0.328 \\
\hline \multirow[t]{6}{*}{ Mean $\pm S D \%$ predicted Forced Expiratory Volume in 1 second } & Baseline & $86.9 \pm 24.8$ & $81.2 \pm 19.9$ & 0.3 \\
\hline & & $\mathrm{n}=7$ & $n=53$ & \\
\hline & End of biologics trial & $91.9 \pm 25.7$ & $82.4 \pm 18.6$ & \\
\hline & & $\mathrm{n}=7$ & $n=53$ & \\
\hline & $\begin{array}{l}\text { Paired comparison of baseline and end } \\
\text { of trial P-value }\end{array}$ & 0.381 & 0.607 & \\
\hline & Median (IQR) change/person & $1(-4-8)$ & $3(-8-12.8)$ & 0.949 \\
\hline \multirow[t]{4}{*}{ Median (IQR) Fractional exhaled Nitric Oxide - parts per billion } & Baseline & $65(18.5-101) n=5$ & $\begin{array}{l}44(21.8-77.8) \\
n=38\end{array}$ & 0.698 \\
\hline & End of biologics trial & $26(15-54) n=5$ & $27(14-50) n=38$ & \\
\hline & $\begin{array}{l}\text { Paired comparison of baseline and end } \\
\text { of trial P-value }\end{array}$ & 0.438 & $0.0499 *$ & \\
\hline & Median (IQR) change/person & $-11(-80-16))$ & $-6.68(-35.2-8.78)$ & 0.755 \\
\hline \multirow[t]{4}{*}{$\begin{array}{l}\text { Median (IQR) Mini-Paediatric Asthma Quality of Life Questionnaire } \\
\text { score }\end{array}$} & Baseline & $6.04(3.56-7) n=8$ & $\begin{array}{l}4.19(3.23-5.42) \\
n=32\end{array}$ & 0.083 \\
\hline & End of biologics trial & $6.69(4.57-7) n=8$ & $\begin{array}{l}6.05(4.11-6.59) \\
n=32\end{array}$ & \\
\hline & $\begin{array}{l}\text { Paired comparison of baseline and end } \\
\text { of trial P-value }\end{array}$ & 0.188 & $0.0008^{* * *}$ & \\
\hline & Median (IQR) change/person & $\begin{array}{l}0.115(0-1.75) \\
n=8\end{array}$ & $\begin{array}{l}0.885(-0.263- \\
2.11) n=32\end{array}$ & 0.550 \\
\hline Proportion of patients with Asthma Control Test or childhood & Baseline & $3(20) n=15$ & $8(14) n=57$ & 0.277 \\
\hline Asthma Control Test $\geq 20$ & End of biologics trial & $5(55.6) n=9$ & $15(34.9) n=43$ & \\
\hline \multirow[t]{2}{*}{ - No. (\%n) } & Change from baseline & 0.0994 & $0.0175^{*}$ & \\
\hline & P-value & & & \\
\hline
\end{tabular}

Normality tested with Shapiro-Wilkes.

Normal data compared with paired t-test, non-normal with Wilcoxon signed-rank and categorical data with Fisher's exact test.

'mepolizumab and omalizumab exacerbation baselines are the 6 and 4 months before treatment initiation respectively.

*denotes $P \leq 0.05$, ** denotes $P \leq 0.001$ and *** denotes $P \leq 0.0001$.

for large-scale paediatric mepolizumab studies, especially given evidence of low Type- 2 cytokines in children with severe asthma.

\section{P203 EFFECTIVENESS OF ANTI-IL4R THERAPY FOLLOWING SUBOPTIMAL RESPONSE TO ANTI-IL5/5R THERAPY IN SEVERE EOSINOPHILIC ASTHMA}

${ }^{1}$ TW Mason, ${ }^{1,2} \mathrm{AP}$ Hearn, ${ }^{1} \mathrm{G}$ d'Ancona, ${ }^{1} \mathrm{M}$ Fernandes, ${ }^{1} \mathrm{C}$ Roxas, ${ }^{1} \mathrm{~L}$ Green, ${ }^{1} \mathrm{~L}$ Thomson, 1J Dhariwal, 'AM Nanzer, ${ }^{1,2}$ DJ Jackson. 'Guy's Severe Asthma Centre, Guy's Hospital, Guy's and St Thomas' NHS Foundation Trust, London, UK; ${ }^{2}$ Asthma UK Centre, King's College London, London, UK

\subsection{6/thorax-2021-BTSabstracts.312}

Introduction Dupilumab is an anti-IL4R monoclonal antibody $(\mathrm{mAb})$ with proven efficacy in severe eosinophilic asthma (SEA). We previously reported that a suboptimal response to the eosinophil-targeting anti-IL5/5R mAbs mepolizumab and benralizumab is seen in $27 \%$ and $14 \%$ of patients with SEA respectively. ${ }^{1,2}$ The mechanism of this is not well-understood. It is unknown whether such patients respond in a clinically meaningful way following a switch to dupilumab.
Methods We performed a retrospective analysis of the clinical effectiveness of dupilumab (minimum 6 months treatment) in patients with SEA at our tertiary severe asthma centre who had failed to adequately respond to at least one of the antiIL-5/5R mAbs. Change in the annualised exacerbation rate (AER), maintenance oral corticosteroids (mOCS) requirements and ACQ-6 was recorded.

Results Twenty-one patients (mean age 43.5, 71\% female, $70 \%$ atopic) were included in the analysis. 9/21(42.9\%) had co-morbid nasal polyposis and $3 / 21(14.3 \%)$ had eczema. The baseline FeNO was 62.5(38-87)ppb. 15/21 were switched from benralizumab (including $7 / 15$ who had previously failed mepolizumab); 5/21 from mepolizumab and 1/21 from reslizumab. 3/21 had previously failed omalizumab prior to a switch to an anti-IL5/5R mAb. Fifteen patients were receiving mOCS at the time of commencing dupilumab.

At six months, the daily median mOCS dose fell from $10 \mathrm{mg}(6.25-22.5 \mathrm{mg})$ to $3 \mathrm{mg}(0-7.5 \mathrm{mg}), \mathrm{P}<0.002$. ACQ-6 improved by 1.04 units from $2.94 \pm 1.31$ to $1.90 \pm 1.40$, $\mathrm{p}=0.037$. There was a trend towards improvement in AER from $1.56 \pm 1.50$ to $0.75 \pm 1.24, p=0.063$. Median blood eosinophil count rose from $0.0(0-0.2)$ to $0.5(0.3-1.2), \mathrm{p}<0.001$. 
One patient discontinued dupilumab during the follow-up period.

Conclusion A minority of individuals with SEA have a suboptimal response to eosinophil-targeted therapy with an anti-IL5/ $5 \mathrm{R} \mathrm{mAb}$. We report significant clinical improvements following initiation with dupilumab suggesting an important role for IL4/-13 in these patients. The exact mechanisms require further research and are likely to lead to a reclassification of T2-high sub-phenotypes.

\section{REFERENCES}

1. Kavanagh JE, d'Ancona G, Elstad M, et al. Real-world effectiveness and the characteristics of a 'super-responder' to mepolizumab in severe eosinophilic asthma. Chest. 2020Aug;158(2):491-500.

2. Kavanagh JE, Hearn AP, Dhariwal J, et al. Real-world effectiveness of benralizumab in severe eosinophilic asthma. Chest.2021;159(2):496-506.

\section{P204 COVID-19 IN THE ABSENCE OF EOSINOPHILS: A CASE SERIES OF CONFIRMED INFECTION WHILST ON TREATMENT WITH BENRALIZUMAB}

${ }^{1}$ CHR Francis, ${ }^{1,2} \mathrm{AP}$ Hearn, 'S Ratnakumar, 'A Taylor, 'J Duckitt, ' $\mathrm{U}$ Ahmed, 'J Dhariwal, ${ }^{1}$ AM Nanzer, ${ }^{1,2}$ DJ Jackson. 'Guy's and St Thomas' NHS Foundation Trust, London, UK; ${ }^{2}$ Asthma UK Centre, King's College London, UK

\subsection{6/thorax-2021-BTSabstracts.313}

Background Blood eosinopaenia was one of the earliest reported findings in hospitalised patients with COVID-19, questioning whether eosinophils could have an anti-viral or deleterious role in the immune response against SARS-CoV2. Benralizumab is an anti-IL5R monoclonal antibody licensed for the treatment of severe eosinophilic asthma (SEA) and causes the near-complete depletion of blood and tissue eosinophils. As such, it offers the opportunity to explore the impact of eosinopaenia at the time of infection on outcome with COVID-19.

Method Patients started on treatment with benralizumab (up until April 2021) for SEA at our regional asthma centre were contacted by telephone throughout May and June 2021 to establish whether they had experienced a confirmed (PCR-positive) SARS-CoV2 infection since commencing benralizumab. Clinical and demographic characteristics were recorded along with the outcome of infection, including the need for hospitalisation or intensive care admission. Patients requiring hospitalisation were compared to those experiencing mild infections.

Results Data on 268 patients treated with benralizumab was collected with 24/268 (9\%) confirming SARS-CoV2 infection with a positive PCR test. Of these 18/24 (75\%) experienced mild infections that did not require hospitalisation. Of the 6/ 24 requiring hospitalisation, the median (IQR) length of stay was 6 (1-8) days. No patients required ICU admission or mechanical ventilation. There was no significant difference in baseline characteristics between hospitalised and non-hospitalised patients. However, it is noteworthy that a higher proportion of hospitalised patients were male $(50.0 \%$ vs $38.9 \%)$ and had a higher mean BMI (32.1 vs 29.5).

Discussion In the context of drug-induced eosinopaenia with benralizumab, $75 \%$ of patients with severe asthma experienced mild COVID-19 disease. This is likely to be an underestimate given that other patients may have experienced an asymptomatic infection or not pursued PCR testing in the context of mild infection. Although caution is needed due to the small sample size, these results do not support a significant role for eosinophils in SARS-CoV2 infection.

\section{P205 \\ ELECTIVE INPATIENT SYSTEMATIC EVALUATION OF DIFFICULT TO TREAT ASTHMA; CASE SERIES DEMONSTRATING THE CLINICAL VALUE AND IMPROVED PATIENTS OUTCOMES}

A Cass, AH Mansur. Univesity Hospitals Birmingham, Birmingham, UK

\subsection{6/thorax-2021-BTSabstracts.314}

Background Whilst the assessment and management of difficult to treat asthma (DTA) is largely conducted in outpatient settings, this seems inadequate for a minority of patients presenting with complex disease and high healthcare utilisation. Structured multidisciplinary inpatient assessment for this group may delineate the main cause/s of their disease and allow planning and effective management strategies.

Aim To assess the Impact of elective admission for assessment and management of difficult to treat asthma patients presenting with frequent hospital admissions and/or excessive oral corticosteroids (OCS) dependency.

Methods Patient who were electively admitted and had multidisciplinary structured inpatient assessment at our tertiary severe asthma centre were considered for this analysis. Demographics and clinical details were retrieved from patients' medical and electronic records including the local dendrite system registry. Standard statistics were applied.

Results We included 21 patients, $81 \%$ females (17/21), mean age 41.6 years (23-73), mean BMI $35 \pm 10 \mathrm{~kg} / \mathrm{m}^{2}$, pre-admission prednisolone dose was $29.1 \pm 15.3 \mathrm{mg} /$ day, mean hospital admissions in the preceding year was $6.9 \pm 5.2$, and the proportion with ITU admission ever was $71.4 \%$.

The mean length of stay was 11.4 days (range 5-21) in which asthma diagnosis was confirmed in $67 \%$ of cases but severe asthma was excluded in the majority (70\%) and only $30 \%$ of patients had high type 2 inflammation. This allowed significant reduction of maintenance prednisolone dose to a mean of $5.6 \pm 2.6 \mathrm{mg} /$ day (figure 1) and $73 \%$ of cases were converted to replacement hydrocortisone. The primary drivers of symptoms were breathing pattern disorder (67\%), inducible laryngeal obstruction (42\%), excessive dynamic airway collapse (42\%), and multiple diagnoses were often observed (median comorbidities 4.5 , 95\%CI 4-5.6).

Conclusions In this study we observed significant benefit to patients in establishing the correct diagnosis, revision of treatment and reduction of dependency on maintenance oral

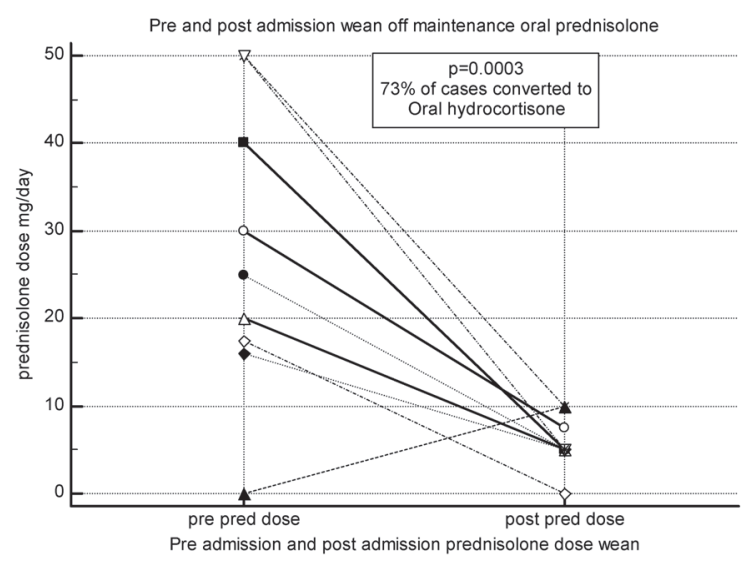

hydrocortisone due to adrenal suppression

Abstract P205 Figure 1 